Brit. J. industr. Med., 1962, 19, 100.

\title{
INVESTIGATION OF AN OUTBREAK OF PHOTODERMATITIS CONFINED TO ONE SHOP IN A LARGE FACTORY
}

\author{
BY \\ D. W. WYNN JONES \\ From Vandervell Products Limited
}

(RECEIVED FOR PUBLICATION DECEMBER 4, 1961)

\begin{abstract}
Out of 106 men employed in a foundry attached to a large factory, 29 men developed a photosensitization dermatitis over the period of three months. Three men out of 1,700 employed in the remainder of the works developed a similar eruption, but no common factor was apparent. Owing to the very high incidence in the foundry, a sensitizer associated with the process was suspected and it seemed likely that it would prove to be an atmospheric contaminant. Many chemical investigations and skin tests were carried out without positive result, though much interesting information regarding combustion products of various materials was obtained.

Some of the men were referred to dermatological clinics in three neighbouring hospitals. In one of these clinics, a similarity was noticed between six cases recently seen there. This suggested that the sensitizer was not confined to the foundry or to the factory. The cause was eventually traced to an additive (tetrachlorsalicylanilide) in a popular brand of toilet soap, and it was evident that the photosensitivity in varying degrees of severity was widespread over the Home Counties, if not much of the country. It had not hitherto been recognized, as mild cases had required little or no treatment, and severe cases were widely disseminated. It is probable that, had it not been for the very high incidence in the foundry, the eruption would have remained unrecognized for much longer. The high incidence in the foundry is attributed to the frequency with which the men washed there, and possibly to some local aggravating factor not yet discovered.
\end{abstract}

The factory engaged in the manufacture of automobile bearings employed some 1,800 men and women. It was divided into three self-contained shops, a foundry (106 employees), a plating shop (142 employees), and a machine shop (1,592 employees). The latter two shops, being for clean processes, had communal washrooms; the foundry had its own wash- and shower-room. Liquid soap was in use throughout the factory, but in the foundry showerroom a popular brand of germicidal toilet soap was also in use, liquid soap being inconvenient for use when showering. Barrier cream was used liberally in the foundry but was banned in many parts of the factory for technical reasons.

In all, 32 cases presented throughout the factory, but 29 were in the foundry where $31 \%$ of employees were affected. This high incidence pointed to a work hazard in this shop, and although the process was said to have remained unchanged for 15 years, subsequent investigation showed that there had been changes in sources of supply of raw materials. It was also possible that there had been changes in the composition of the oils and lubricants used, and in the laundering process of the overalls.

The foundry (Fig. 1) is a large well-lit building in which coils of steel strip are stored and moved as required by diesel fork lifts; they are then butted and spot welded into one continuous length which passes through a degreaser into a silicone carbide preheater and thence into a gas furnace where it is heated to $2000^{\circ} \mathrm{F}$. in an inert atmosphere consisting of nitrogen, $\mathrm{CO}_{2}$, and $\mathrm{CO}$, and some hydrogen. In this atmosphere, which acts as a flux, bronze at $2000^{\circ} \mathrm{F}$. is poured on from an electric induction melting furnace with gas-heated pouring nozzles. The strip is cooled in quenching oil, then coiled, and finally milled, polished, slit, and recoiled.

The foundrymen work round the clock in 12hour shifts, and most have worked in this shop for at least six years. The work is oily and dirty, particularly near the furnaces. The quenching oil sometimes ignites with the production of much smoke, fume, 


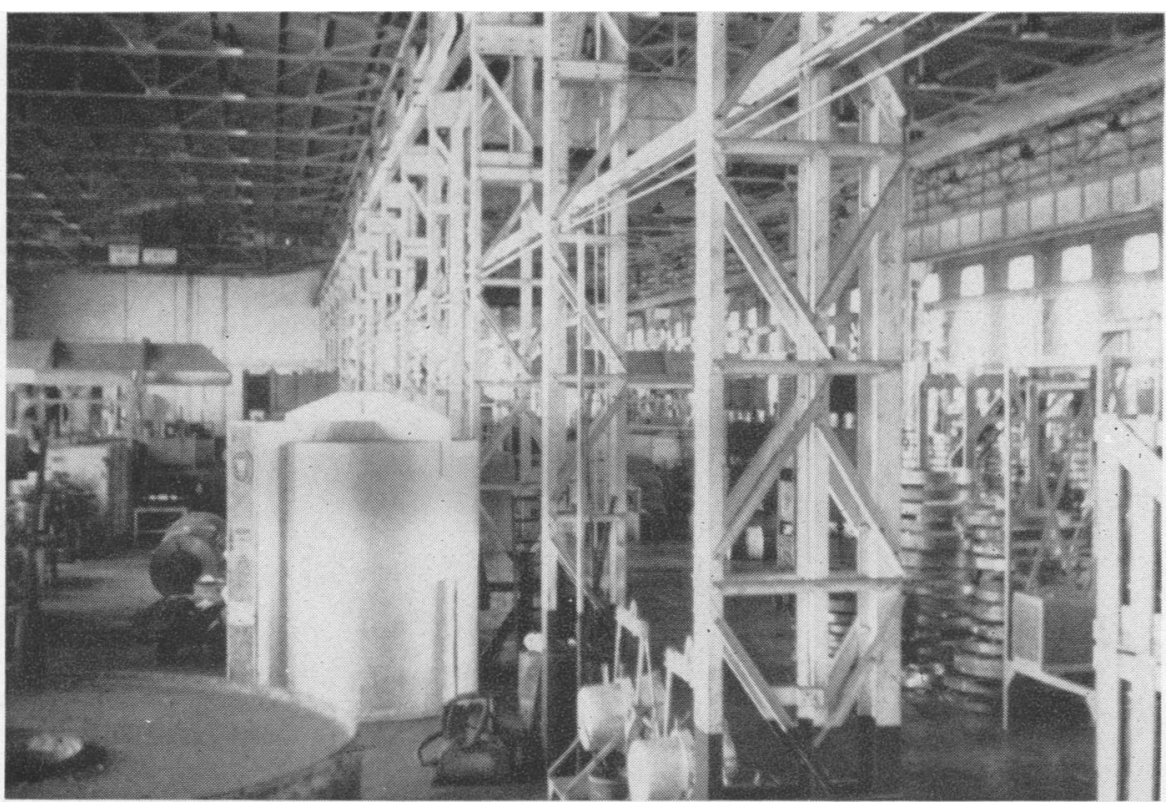

FIG. 1.-View of part of shop. The furnace cowlings are seen on the left.

and heat. The men wear a uniform of absorbent sweat shirt and trousers, which gets very dirty and presents laundering problems necessitating the use of powerful cleansers. The men may wash as many as 12 times a shift in order to keep clean.

The outbreak started in October 1960, with one or two cases reporting each week, reaching a maximum of six new cases on November 7, 1960. The eruption was nearly always preceded by a deep burning sensation in the skin of the dorsum of the hands or the exposed areas of the face and neck, and sometimes on both these areas. Erythema then

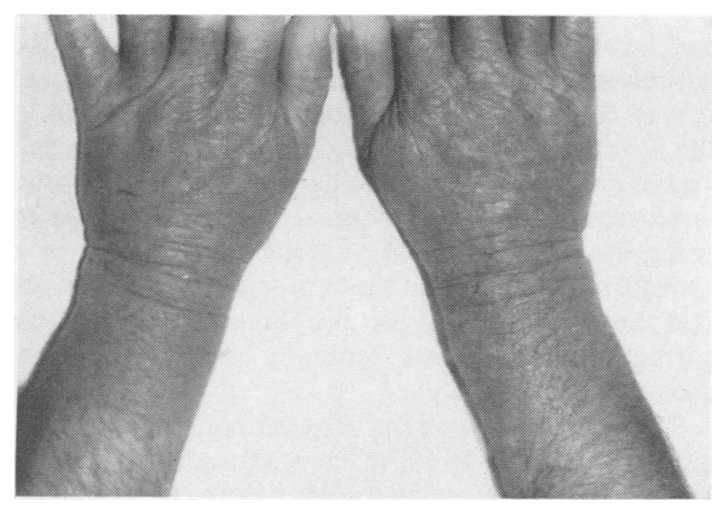

Fig. 2.-Subacute stage of exposed areas of hands and forearms. developed swiftly progressing in severe cases to exudation, and finally desquamation, leaving the skin dry and tight with the appearance of lichenification, and sometimes cracking and pigmentation. The course was swift, clearing in some cases in as little as three to four days. Men mildly affected frequently complained only of burning with transient erythema. The conditions of some, however, particularly those with lichenification and cracking, became chronic, lasting two or three months with remissions and exacerbations, the latter often being provoked by bright weather conditions. Secondary infection was sometimes present, especially on the neck. There was never any interdigital involvement, the dorsum of the hands and wrists only being involved (Fig. 2). On the face the rash was most marked on the prominences, the malar regions, tips and lobes of ears, nose, and chin, and the sides and back of neck. Cracking of the lips was fairly common. There was always a sharp line of demarcation at the neck and collar line (Figs. 3 and 4).

It was clear that the rash affected only exposed parts. It was also observed that men reported most frequently on a Monday morning after a week-end away from work. One particular Monday in November produced no less than six new cases, whilst after the long Christmas week-end a further five recurrences presented. Inquiries revealed that most new cases and recurrences presented after 


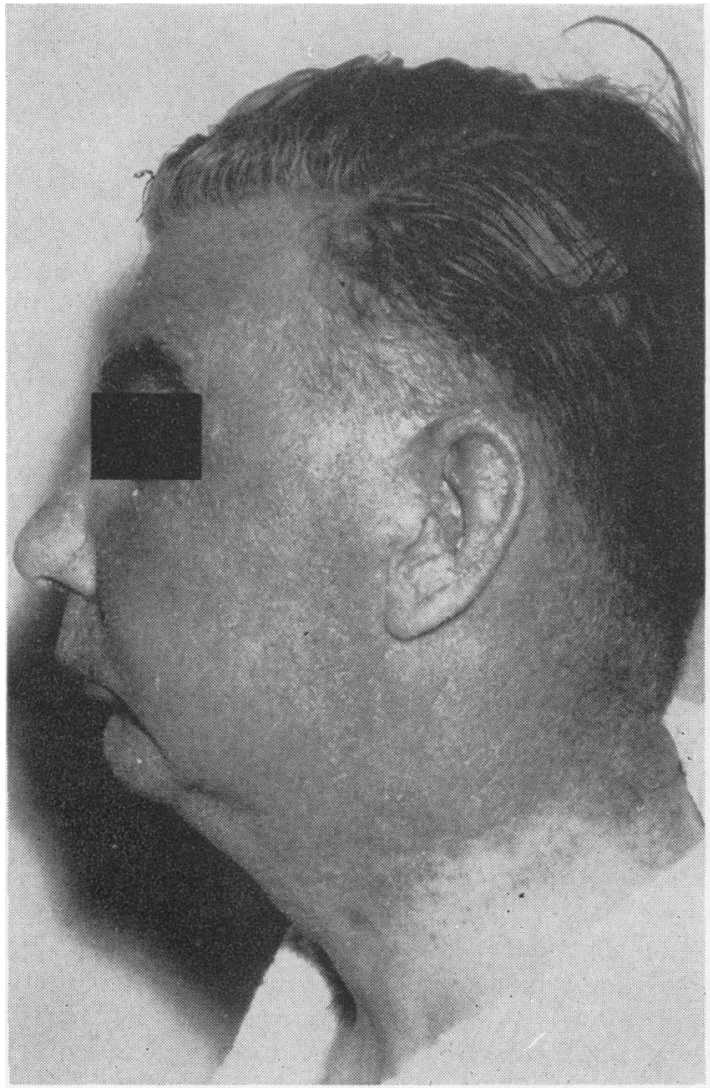

Fig. 3.-Side view of acute stage showing demarcation at collar line.

bright and sunny week-ends, and also that the persons affected had spent their week-end in the open, watching sport or in the garden; two had driven open-top cars. It was therefore assumed that the dermatitis was caused by a photosensitizing agent. It was thought at first that the sensitizer was unconnected with the process which had been unchanged for 15 years, many of the men having worked on it for much of that time. The manufacturers of the barrier cream and the liquid soap were approached and asked if any alteration had been made in the manufacture of their products. Both these substances were available in the remainder of the factory, but, owing to the dirty nature of their work, the foundrymen used about 10 times as much of these products per head, as workers in other parts of the factory. Both these products were also made by small manufacturers and were for this reason suspect. Despite the manufacturers' assurances both these substances were withdrawn.

By this time a typical case occurred in an electrician

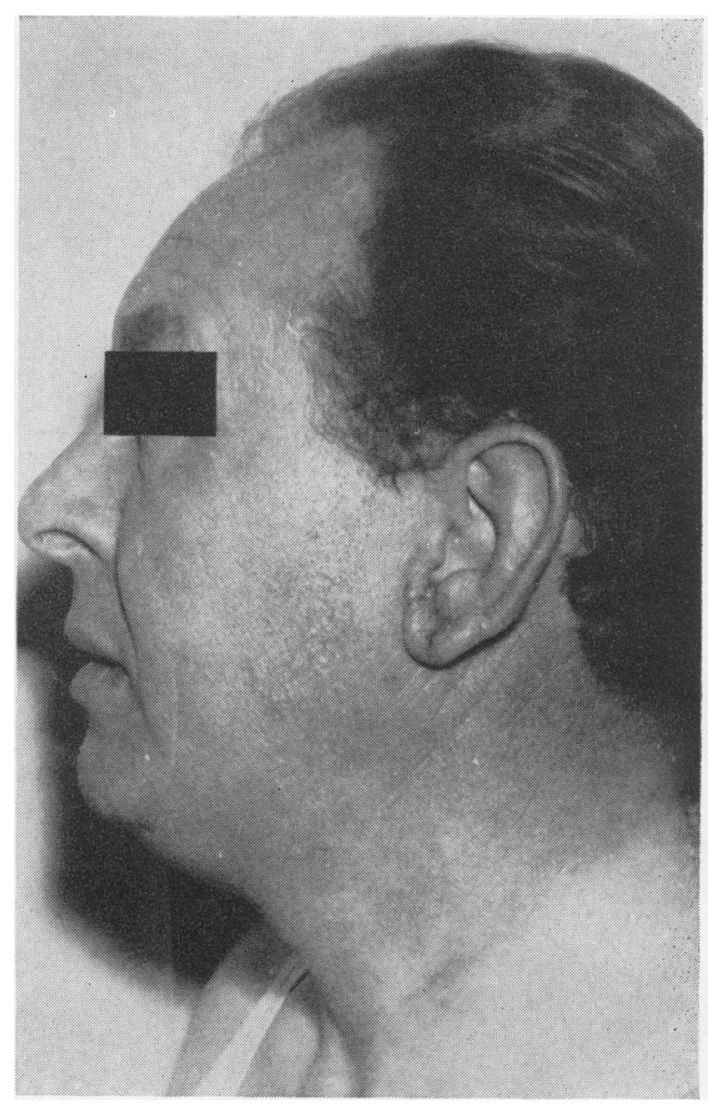

FIG. 4. Subacute stage, showing "leathering" thickening and lichenification. Also note demarcation line at neck.

in the plating shop, and two weeks later another electrician in the plating shop became affected. Neither of these two men had any connexion with the foundry, nor did they use its washrooms. Clinically there appeared to be a common factor but no satisfactory explanation was then found. There had been no improvement in the situation since the withdrawal of the liquid soap and barrier cream, and it was decided to investigate possible sensitizers in the foundry. Those who had been severely affected, and in particular those who had suffered recurrences, were removed from the foundry and forbidden to enter its precincts or its washrooms. They were also forbidden to wear the uniform, lest a sensitizer had been introduced in the laundering of these garments. These men generally made good progress.

The occupations of the affected men were investigated and found to be diverse, though there was a tendency for more cases to come from the vicinity of the furnaces, where they worked in conditions of 
considerable heat, dirt, and atmospheric contamination. The asbestos and P.V.C. gloves were investigated, as were the rust-preventing substances and the oils and lubricants used in the process. Patch tests with these substances were undertaken, as with the liquid soap and barrier cream, and with washings from clean and dirty overalls, but no positive results were obtained.

Some men said that their skin tingled on entering the foundry, and others that the rash started immediately they came to work on a Monday morning. This focused attention on a possible atmospheric contaminant. The main source of fume seemed to be when copper scrap was fed to the furnaces. The source of this scrap had changed, the material in use at the time being copper wire, the insulation of which had been incompletely burned off, leaving a considerable quantity of semicarbonized residue. The insulating materials used on such conductors include paper, dry, or impregnated with resins, plastics, and compounded mineral oils; cotton, dry or impregnated; bitumen; lead, etc. Pyrolysis of this residue under conditions similar to those in the furnaces gave a very complex mixture of products in varying physical states. These products included phenols, primary and substituted amines, carboxylic acids, olefins, aromatic hydrocarbons, and carbon-black. Further investigation into this interesting field was abandoned when the cause of the dermatitis was found elsewhere. There was also considerable fume in the region of the quenching bath as the hot strip passed through it. The quenching oil was a clear medium viscosity naphthonic-based oil without additives, which became darker in colour and changed in smell after use. Samples of the oil before and after use were subjected to chromatographic analysis to separate out aromatic hydrocarbons. After use there was an appreciable increase in the amount of aromatic hydrocarbons present, and a change in fluorescence from blue to bright yellow. The fumes around the quenching bath contained carbonaceous particles and mineral oil mist. From the materials examined it was evident that the atmosphere in the vicinity of the furnaces and quenching bath was contaminated with aromatic hydrocarbons, phenols, and a variety of other noxious materials. Ethereal extracts of this atmosphere when used for patch testing failed to yield a positive result.

After two months some 13 cases had recurred, some more than once. These recurrences, almost invariably associated with a sunny week-end, were often more severe than the original outbreak, and were associated with much oedema of the face and eyelids. Two of these cases had to be admitted to hospital as emergencies. Here they came under the

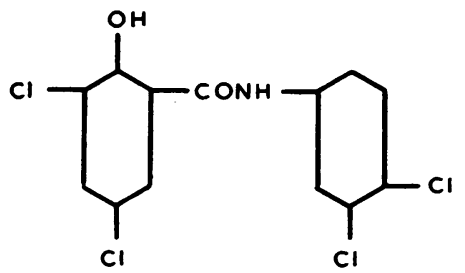

TETRACHLORSALICYLANILIDE

care of Dr. D. S. Wilkinson who informed me that he had seen six similar cases in neighbouring skin clinics. He kindly agreed to help in the investigation and observed that the foundry shower-room was liberally supplied with bars of a popular brand of toilet soap, which, as has already been mentioned, was used only in this washroom. Suspicion had not been focused on this soap, as it was one of the best known germicidal toilet soaps and widely used.

During the ensuing investigation it was observed that a solution of this soap was fluorescent; later it was found that a $1 \%$ solution of the soap was the only substance so far found to give even an equivocal result on patch testing. By this time another employee outside the foundry developed a typical eruption, making now a total of three having no connexion with the foundry. On inquiry it was found that all three used this same brand of toilet soap at home. A common factor was now found. Use of the soap was discontinued and no new cases reported, though recurrences continued to be observed in those who had been severely affected. Some of these recurred sporadically throughout the following summer, some nine months after the initial outbreak.

Inquiries from the manufacturers of the soap revealed that a new germicide, tetrachlorsalicylanilide (T.C.S.A.) had been incorporated in the soap recently in $0.25 \%$ dilution. A limited consumer trial had been conducted with no adverse reports. Subsequent inquiries showed that the trial had been conducted in an industrial area in the North of England during a prolonged period of dull weather. The soap was marketed in July 1960, but had actually been withdrawn just before this outbreak.

Patch tests with a $1 \%$ soap solution were positive in the affected men, and in some cases were aggravated by irradiation with ultraviolet light. Similar tests with the same soap without T.C.S.A. were negative, while tests with $0.25 \%$ T.C.S.A. followed the same pattern as with the affected soap (Fig. 5). "Dab" tests with $1 \%$ T.C.S.A. on the cheek were positive (Fig. 6).

It soon became apparent that the outbreak was widely disseminated throughout the community. 


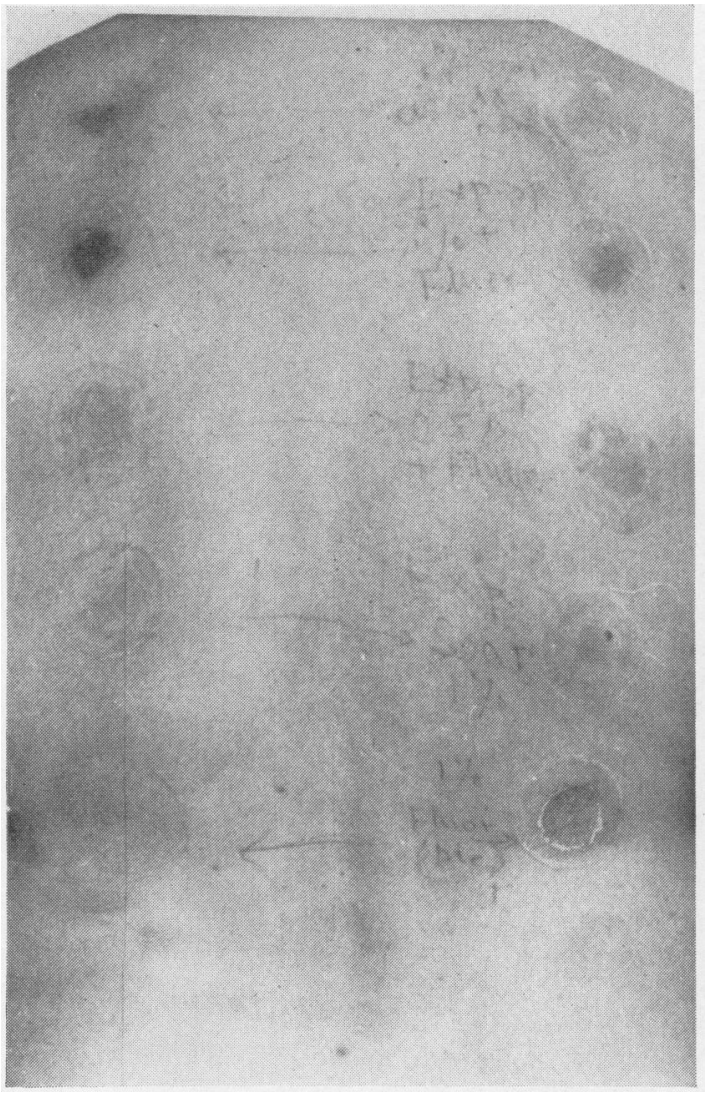

Fig. 5.-Patch tests after 48 hours. Both sides are similar, but the right has been irradiated with a sub-erythema dose of U.V.L. The marked reaction of $1 \%$ T.C.S.A. ("Fluor") both in soap and in alcoholic solution, is seen in the top two, and the bottom patch.

Dr. Wilkinson collected a further 16 cases in his clinics, and $I$ encountered nine clear-cut cases in general practice, both within a period of some six weeks. This was pointed out to the manufacturers who had withdrawn the soap three months previously. They then went to considerable lengths to ascertain that all bars of this type of soap were withdrawn from individual shops.

My thanks are due to Dr. D. S. Wilkinson who observed the similarity between my cases and those in his clinic in High Wycombe, and who finally identified the sensitizer, and who also kindly allowed me to publish

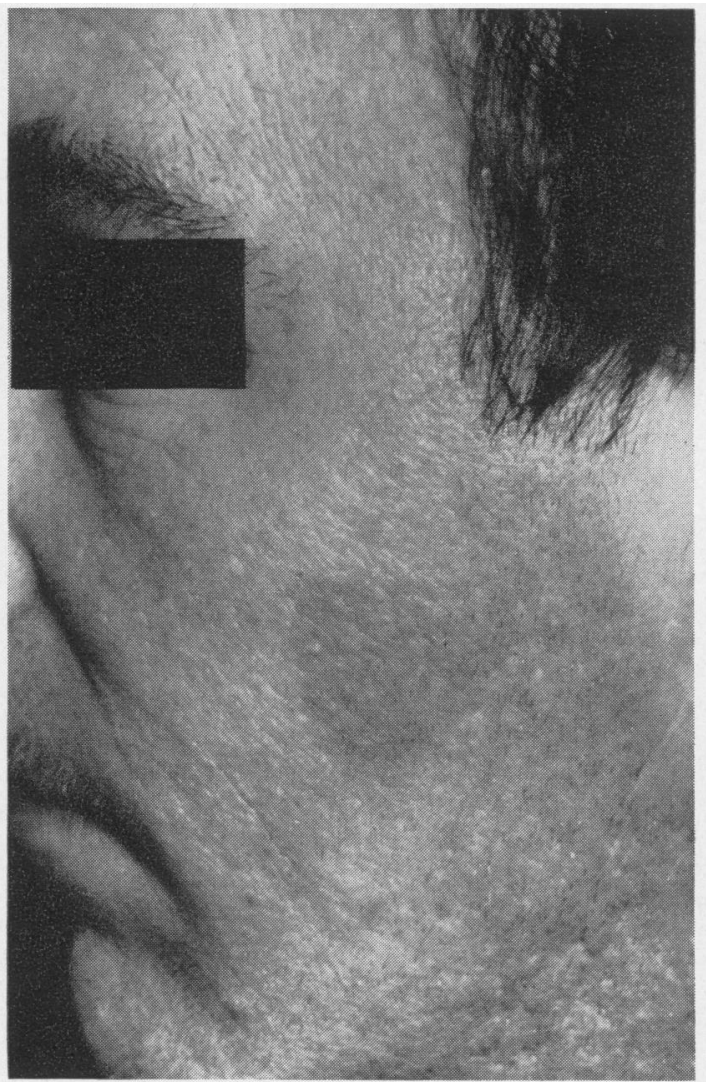

FIG. 6.-Dab tests on cheek with $1 \%$ T.C.S.A. show a positive reaction.

his photographs of cases; to the Slough Occupational Hygiene Service for assistance in extracting atmospheric contaminants, examining the ventilating system, and undertaking pyrolysis of the scrap; and to Dr. D. M. Trott, H.M. Medical Inspector of Factories, whose hunch that the sensitizer was not connected with the process subsequently proved correct.

\section{BIBLIOGRAPHY}

Lennon, W. J., Furia, T. E., and Zussman, H. W. (1960). Soap, Perfum. Cosm., 33, 609.

Wilk (1960). Soap Chem. Spec., 36, No. 3, p. 51 .

Wilkinson, D. S. (1961). Brit. J. Derm. 73, 213.

- (1961). Proc. roy. Soc. Med., 54, 817. 